\title{
FGFRL1 deficiency reduces motility and tumorigenic potential of cells derived from oesophageal squamous cell carcinomas
}

\author{
YOSHINORI TAKEI $^{1}$, TAKAFUMI MATSUMURA ${ }^{2}$, KAZUAKI WATANABE $^{1}$, \\ HIROKAZU NAKAMINE ${ }^{3}$, TETSUO SUDO ${ }^{1}$, KAZUHARU SHIMIZU $^{1}$ and YUTAKA SHIMADA ${ }^{1}$ \\ Departments of ${ }^{1}$ Nanobio Drug Discovery and ${ }^{2}$ Genomic Drug Discovery Science, \\ Graduate School of Pharmaceutical Science, Kyoto University, Kyoto 606-8501; ${ }^{3}$ Division of \\ Pathology and Laboratory Medicine, The Japan Baptist Hospital, Kyoto 606-8273, Japan \\ Received January 17, 2018; Accepted April 16, 2018
}

DOI: $10.3892 / \mathrm{ol} .2018 .8739$

\begin{abstract}
Oesophageal squamous cell carcinoma (ESCC) is an aggressive cancer that resulted in $\sim 400,000$ mortalities worldwide in 2012. It was reported previously that fibroblast growth factor receptor-like 1 (FGFRL1) is highly expressed in ESCC patients with lymph node metastasis and poor prognosis accordingly. FGFRL1 is an FGFR that lacks tyrosine kinase activity, whereas the activity is critical for other FGFRs to activate intracellular signalling. The mechanism by which FGFRL1 promotes the aggressiveness of ESCCs is unknown. In the present study, two independent FGFRL1-deficient cell lines were generated from human ESCC KYSE520 cells, in order to investigate the relationship of FGFRL1 with the aggressiveness of ESCCs. FGFRL1-deficiency did not affect proliferation of KYSE520 cells in vitro. However, a xenograft mouse model demonstrated that FGFRL1-deficiency decelerated tumour growth in vivo. The haematoxylin-eosin staining identified that FGFRL1-deficient cells formed well-differentiated squamous cell carcinomas, whereas wild-type cells formed moderately differentiated squamous cell carcinomas. Microarray analysis of mRNA expression revealed that FGFRL1-depletion resulted in decreased expression of proteins associated with motility and invasion of tumour cells, matrix metalloproteinase-1 and fibroblast growth factor binding protein 1. The wound-healing assay indicated that depleting FGFRL1 reduced cell motility. Furthermore, the invasiveness of FGFRL1-deficient cells was lesser than that of wild-type KYSE520 cells. In the FGFRL1-deficient KYSE520 cells, actin filaments around the nucleus were observed sparsely, whereas the filaments along
\end{abstract}

Correspondence to: Dr Yoshinori Takei, Department of Nanobio Drug Discovery, Graduate School of Pharmaceutical Science, Kyoto University, 46-29 Shimoadachicho, Yoshida, Sakyo-ku, Kyoto 606-8501, Japan

E-mail: ytakei@pharm.kyoto-u.ac.jp

Key words: migration, metastasis, oesophageal squamous cell carcinoma the plasma membranes were observed as frequently as those in the parent KYSE520 cells. These results demonstrate that FGFRL1 may be involved in regulation of protein expression, actin filament assembly and tumorigenic potential of ESCC cells.

\section{Introduction}

Oesophageal cancer is the sixth leading cause of cancer-associated mortality behind lung, liver, gastric, colorectal, and breast cancer, worldwide in 2012 (1). In total, 400,000 mortalities were attributed to the disease worldwide in 2012 (2). The cancer includes two main sub-types; oesophageal squamous-cell carcinoma (ESCC) and oesophageal adenocarcinoma. While oesophageal adenocarcinoma is widespread in Europe and the USA (2), ESCC is diagnosed more often in Asia, Africa and South America (3). In Japan in 2009, 90.5\% of oesophageal cancers were squamous-cell carcinomas (4). Cisplatin is used in combination with fluorouracil as first-line therapy, followed by docetaxel or paclitaxel as second-line therapy to treat oesophageal cancer in Japan (5). No treatment strategy has been defined for patients with oesophageal cancer who are refractory or intolerant to the standard therapies.

Fibroblast growth factor receptor-like 1 (FGFRL1) belongs to the FGFR protein family (6). It has an extracellular FGF-binding site, which is highly homologous with the other members of the family (7). In contrast to other FGFRs, the intracellular domain of FGFRL1 lacks the tyrosine kinase domain; rather, it comprises the tandem tyrosine-based motif and the histidine-rich region (8). Mice lacking FGFRL1 expression exhibit malformation of the metanephros $(9,10)$ and the diaphragm $(11,12)$, and therefore succumb at birth. Thus, FGFRL1 serves an essential function in normal development of the kidney and the diaphragm. However, the mechanism by which FGFRL1 contributes to the development of those tissues remains unresolved.

The authors of the present study reported previously that transient inhibition of FGFRL1 expression induces cell cycle arrest and apoptosis of ESCC cells (13). Expression of FGFRL1 tends to associate with lymph node metastasis; therefore, high expression of FGFRL1 in ESCCs suggests poor prognosis of patients (14). FGFRL1 in ESCC cells is frequently co-expressed 
with either FGFR1 or FGFR4, and an in situ proximity ligation assay indicated that FGFRL1 forms a hetero-dimer with FGFR1 or FGFR4 (15). These results suggest that FGFRL1 serves a function in the aggressive behaviour of ESCCs.

Consistent with these results in ESCCs, downregulation of FGFRL1 expression decreases proliferation of head and neck squamous carcinoma cells (SCC10A) (16). Furthermore, ovarian tumours exhibit aberrant expression of FGFRL1 (17), and FGFRL1 mutation is observed frequently in colorectal tumours (18). Taken together with the results of the authors' previous study, the data in these studies suggest that FGFRL1 serves an important function in cancer generation and/or expansion.

In the present study, cell lines deficient for FGFRL1 expression were established from KYSE520 ESCC cells, in order to investigate the function of FGFRL1 in ESCC cells. FGFRL1 deficiency decreased cell motility and tumour growth in a mouse xenograft model.

\section{Materials and methods}

Materials. Anti-actin (cat. no. ab8226), anti-fibroblast growth factor binding protein 1 (FGFBP1) (cat. no. ab215353) and anti-matrix metalloproteinase (MMP)-1 (ab38929) antibodies were purchased from Abcam (Cambridge, UK). Anti-FGFRL1 (AAB1403271) was purchased from Merck KGaA (Darmstadt, Germany). Alexa Fluor 488-labelled phalloidin (8878) was purchased from Cell Signalling Technology, Inc. (Danvers, MA, USA).

Cell culture and genetic depletion of the FGFRL1 gene using clustered regularly interspaced short palindromic repeats (CRISPR)-cas9. The KYSE520 ESCC cell line was previously established from human ESCC by the authors (19). KYSE520 cells were maintained on collagen I-coated plates in Ham's F12/RPMI-1640 medium (Thermo Fisher Scientific, Inc., Waltham, MA, USA) containing 5\% fetal bovine serum (FBS; Thermo Fisher Scientific, Inc.). For genetic depletion of the FGFRL1 gene, KYSE520 cells were cotransfected with tracrRNA, a plasmid encoding Cas9 (GE Healthcare, Chicago, IL, USA) and crRNA (Fasmac, Inc., Kanagawa, Japan) for the FGFRL1 gene (5'-CAGGGGGCUCGGCGUCAUCUGUUU UAGAGCUAUGCUGUUUUG-3') using Lipofectamine 3000 (Thermo Fisher Scientific, Inc., Waltham, MA, USA). At $24 \mathrm{~h}$ after the transfection, the cells were harvested with trypsin and seeded in $10 \mathrm{~cm}$ diameter cell culture dishes at a density of $2 \times 10^{4}$ cells/dish. Following overnight culture, the medium was changed to Ham's F12/RPMI-1640 containing 5\% FBS and $2 \mu \mathrm{g} / \mathrm{ml}$ puromycin and the cells were cultured for 3 days. The cells were then cultured in Ham's F12/RPMI-1640 containing 5\% FBS without puromycin until colonies were visible. Each colony was isolated and cultured separately. In order to identify FGFRL1-/- cells, genomic DNA was prepared and used as a template for PCR (forward primer: 5'-CTCCCA GTTCCACGTGTTAGTGACG-3' and reverse primer; 5'-CGC CAGAACTCACCTC-3'). The thermocycling procedure for PCR included $2 \mathrm{~min}$ at $98^{\circ} \mathrm{C}$, followed by 23 cycles of $30 \mathrm{sec}$ at $97^{\circ} \mathrm{C}, 30 \mathrm{sec}$ at $58^{\circ} \mathrm{C}$ and $1 \mathrm{~min}$ at $72^{\circ} \mathrm{C}$. Ex taq (Takara Bio, Inc., Otsu, Japan) was used for amplification. The PCR products were directly sequenced.
KYSE520 cell xenografts. All mice were handled and cared for in accordance with the Guide of Care and Use of Laboratory Animals, and all experiments were approved by the Ethics Committee of Experimental Animals of Kyoto University (Kyoto, Japan). All surgical procedures and postoperative care regimes were reviewed and approved by the Animal Care and Use Committee of Kyoto University. Wild-type and FGFRL1-deficient KYSE520 cells were harvested with trypsin and resuspended in Matrigel (Corning Incorporated, Corning, NY, USA) at a concentration of $1.5 \times 10^{7}$ cells $/ \mathrm{ml}$. Next, $0.2 \mathrm{ml}$ $\left(3 \times 10^{6}\right.$ cells) of the cell suspension was subcutaneously injected into immunodeficient athymic Balb/c Slc-nu/nu mice (male, 7 weeks old; n=9; Japan SLC, Inc., Nishi-ku, Japan). The mice were maintained on a 12-h light-dark schedule and given ad libitum access to food and water. After 0,2 and 4 weeks major and minor axes of tumours were measured and tumour mass was calculated using the formula (major axis) $\mathrm{x}$ (minor axis) $)^{2} / 2$. As humane endpoints, two conditions were set. If the major axis of the tumour exceeded $20 \mathrm{~mm}$, the experiment ended. If animals lost their weight $>15 \%$ compared with their age-matched control animals, they were also removed from experiments. However, neither of these instances occurred in the present study. At the conclusion of the experiment, only single tumours were observed, and the maximum tumour volume observed in the present study was $1,734.1 \mathrm{~mm}^{3}$.

Western blot analysis. Cells were harvested with trypsin and homogenised in a buffer containing $50 \mathrm{mM}$ Tris- $\mathrm{HCl}(\mathrm{pH} 7.8)$, $150 \mathrm{mM} \mathrm{NaCl}, 1 \mathrm{mM}$ EDTA, $0.5 \mathrm{mM}$ EGTA, $2 \mathrm{mM}$ DTT, 0.5\% TritonX-100, 2X PhosSTOP phosphatase inhibitor mix (Roche Applied Science, Penzberg, Germany) and 1x Complete Protease Inhibitor Cocktail (Roche Applied Science). Following centrifugation at $15,000 \mathrm{x} \mathrm{g}$ for $15 \mathrm{~min}$ at $4^{\circ} \mathrm{C}$, the supernatant was used as the cell extract. Protein concentrations of the cell extracts were assessed using the Bradford method. Proteins $(40 \mu \mathrm{g})$ were separated by SDS-PAGE and then blotted onto a polyvinylidene difluoride membrane. The membrane was incubated with blocking buffer containing 5\% skimmed milk and $0.05 \%$ Triton X-100 in PBS for $1 \mathrm{~h}$ at room temperature, followed by the aforementioned primary antibodies in blocking buffer overnight at $4^{\circ} \mathrm{C}$ and a POD-labelled secondary antibody matched to the first antibody (1:2,000 dilution; cat. no. 7074 for anti-rabbit IgG and 7076 for anti-mouse IgG; Cell Signalling, Inc.) for $1 \mathrm{~h}$ at room temperature. Bound antibodies were visualised using the ECL-Plus reagent (GE Healthcare). Dilutions of primary antibodies for this study were as follows; anti-FGFRL1 $(1: 1,000)$, anti-actin $(1: 2,000)$, anti-FGFR1 (1:100), anti-FGFR3 (1:200), anti-MMP-1 (1:500) and anti-FGFBP1 (1:500).

Phalloidin staining. Cells were cultured at $37^{\circ} \mathrm{C}$ with $10 \% \mathrm{CO}_{2}$ on a plastic coverslip coated with collagen I (Sumitomo-Bakelite Co., Ltd., Tokyo, Japan). For FGF2 treatment, cells were incubated in Ham's F12/RPMI-1640 medium without serum for $5 \mathrm{~h}$ at $37^{\circ} \mathrm{C}$, after which the medium was changed for Ham's F12/RPMI-1640 with or without $20 \mathrm{nM}$ FGF2 and $50 \mu \mathrm{g} / \mathrm{ml}$ heparin (Thermo Fisher Scientific, Inc.). Following FGF2 treatment, cells were fixed with $4 \%$ paraformaldehyde and $0.5 \%$ Triton X-100 in PBS for $30 \mathrm{~min}$ at $4^{\circ} \mathrm{C}$, and incubated with $0.15 \mathrm{M}$ phalloidin-Alexa fluor 488 in PBS for $3 \mathrm{~h}$ at $4^{\circ} \mathrm{C}$. Images were taken using an Olympus Fluo View 


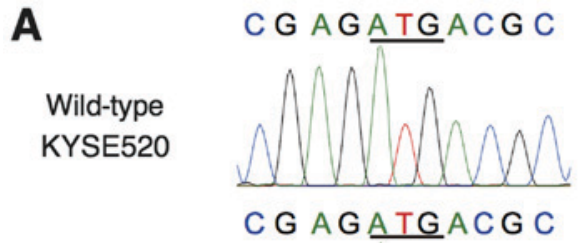

Clone 1

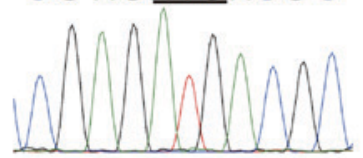

B

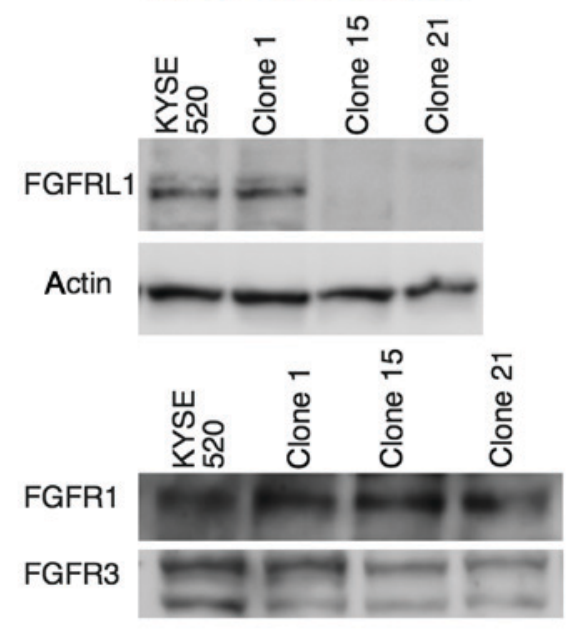

Clone 15
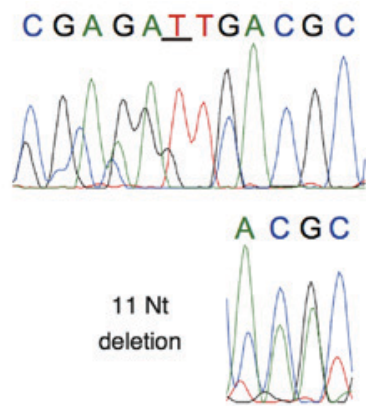

D

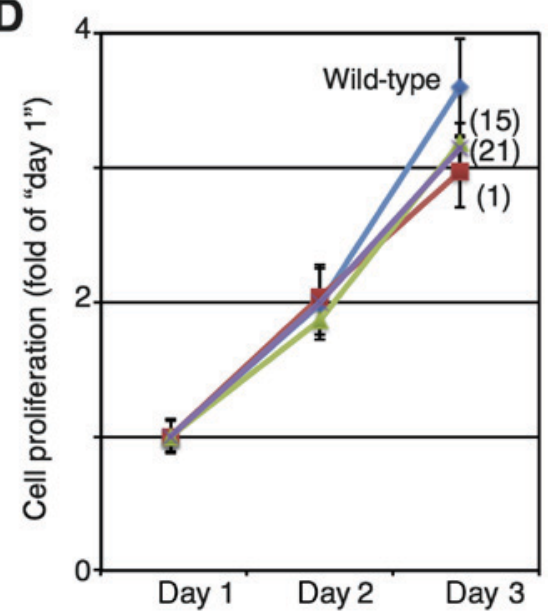

Figure 1. FGFRL1-deficient cells. (A) The sequence of the target site in the FGFRL1 gene. Following transfection of plasmid encoding Cas9 and RNAs, colonies were isolated and the sequences of the FGFRL1 gene were examined. No mutation was detected in the clone 1. A single nucleotide (thymidine) insertion was detected at the first ATG within the FGFRL1 gene in the clone 15. Deletion of 11 nucleotides, including the first ATG within the FGFRL1 gene, was observed in the clone 21. (B) Western blot analysis of KYSE520 cells. Whole cell extracts prepared from each cell line were analysed using SDS-PAGE. (C) Expression of FGFRs in wild-type and FGFRL1-deficient KYSE520 cells. Whole cell extracts prepared from each cell lines were analysed by SDS-PAGE. (D) Proliferation of cells. At the indicated times, cells were harvested with trypsin and counted. Data represent the mean of three experiments. Error bars represent the standard error of the mean. FGFRL1, fibroblast growth factor receptor-like 1; FGFR, fibroblast growth factor receptor; Nt, nucleotide residue.

1000 confocal microscopy system (Olympus Corporation, Tokyo, Japan) with x40 magnification.

Cell migration assay. An i-bidi culture insert (ibidi, Inc.) was set in a $35 \mathrm{~mm}$ cell culture dish. To assess cell migration, cells $\left(5 \times 10^{4}\right)$ were seeded into a well of an i-bidi culture insert with Ham's F12/RPMI-1640 containing 5\% FBS. Following overnight culture at $37^{\circ} \mathrm{C}$, the insert was removed and the cells were washed with F12/RPMI-1640 medium once, and then cells were cultured at $37^{\circ} \mathrm{C}$ in F12/RPMI-1640 medium without serum for $7 \mathrm{~h}$. Cells were observed with an Olympus IX-70 microscopy at x10 magnification.

Cell invasion assay. The CytoSelect 24-well cell invasion assay kit (Cell BioLabs, Inc., San Diego, CA, USA) was used to assess the invasion ability of cells. Cells $\left(1.2 \times 10^{5}\right)$ were seeded on the culture insert (included in the kit) with Ham's F12/RPMI-1640 containing 5\% FBS and incubated for $24 \mathrm{~h}$. Quantification of the invading cells was performed according to the manufacturers protocol. Briefly, after removal of non-invasive cells, invasive cells adhered to the bottom membrane of the insert were quantified using a colorimetric plate reader according to the manufacturers protocol.

Microarray. Total RNA was extracted from $6 \times 10^{6}$ cells of wild-type and FGFRL1-deficient KYSE520 cells, respectively, with Isogen (Nippon gene, Inc., Japan). RNA was amplified with Amino Allyl MessageAMP II kit (Thermo Fisher Scientific, Inc.) and coupled with Cy5 dye with Cy5 Mono-Reactive Dye Pack (GE healthcare), and then hybridized to a human Oligo chip ( $25 \mathrm{k}$; Toray Industries, Inc., Tokyo, Japan) according to the manufacturers protocol.

Statistical analysis. Data are presented as mean \pm standard error of the mean. Statistical analysis was performed using a one-way analysis of variance with Microsoft Excel for Mac 2011 (version 14.7.7; Microsoft Corporation, Redmond, WA, USA). As a post hoc test, Dunnett's test was used. $\mathrm{P}<0.05$ was considered to indicate a statistically significant difference. The Tukey-Kramer method was used to compare tumour mass with the wild type mice. $\mathrm{P}<0.01$ was considered to indicate a statistically significant difference.

\section{Results}

Mutagenesis of FGFRL1 gene. Two cell lines deficient for FGFRL1 expression, clones 15 and 21, were established from an ESCC cell line (KYSE520) using the CRISPR-Cas9 method (Fig. 1A). In the clone 15 line, a single thymidine was inserted into the first ATG site of the FGFRL1 gene. A total of 11 nucleotides (AGGCCGAGATG, which includes the first ATG site of FGFRL1) were deleted from the clone 21. Clone 1 was also 


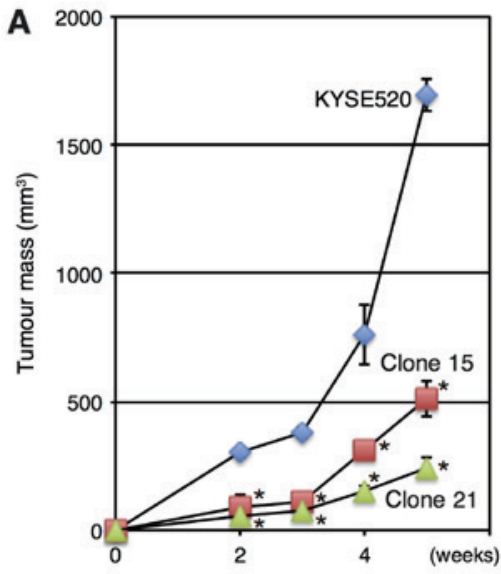

C

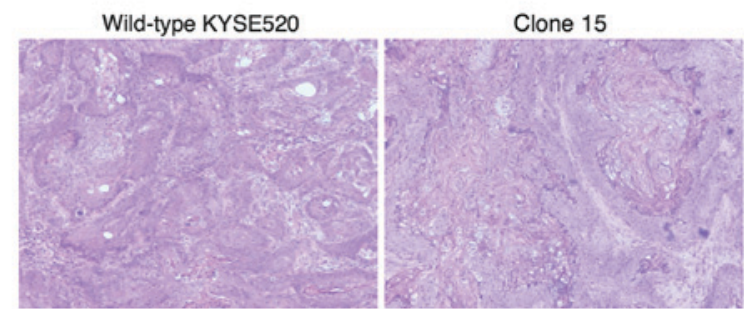

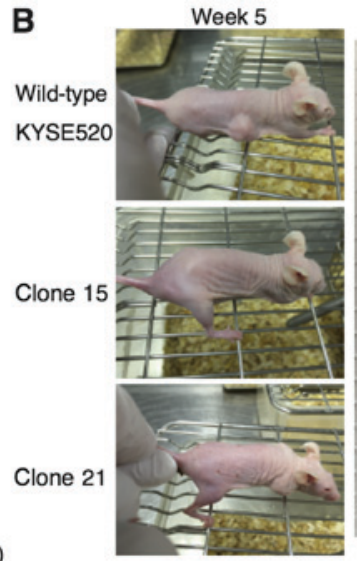

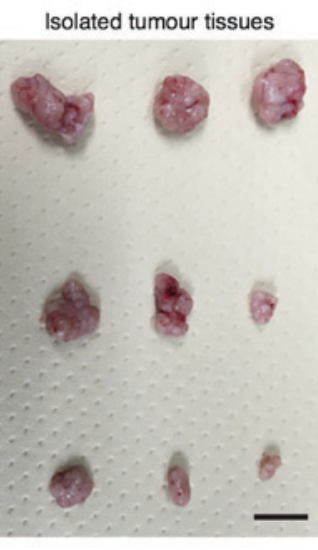

Clone 21

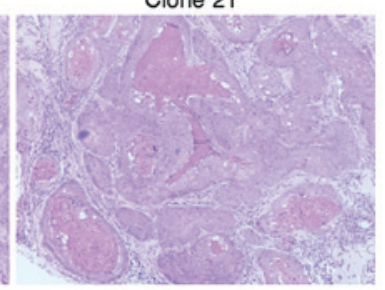

Figure 2. Effects of FGFRL1-deficiency on tumour growth in a xenograft model. (A and B) Following subcutaneous injection of KYSE520 cells, mice were observed at the indicated time points. Wild-type and FGFRL1-deficient KYSE520 cells were each injected into three mice, and tumour mass was estimated. The graph indicates the average values, and the error bars represent the standard error of the mean. ${ }^{~} \mathrm{P}<0.01$ (Tukey-Kramer method) vs. the wild type. Scale bar, $10 \mathrm{~mm}$. (C) Haematoxylin and eosin staining of tumours formed by wild-type or FGFRL1-deficient KYSE520 cells. Tissues were observed using a x20 objective lens. FGFRL1, fibroblast growth factor receptor-like 1.

established from KYSE520 cells treated by the CRISPR-Cas9 method; however, the method failed to produce a mutation at the FGFRL1 gene. No expression of FGFRL1 was detectable in clones 15 and 21, however, its expression was detected in samples derived from parental KYSE520 and clone 1 (Fig. 1B). KYSE520 cells expressed FGFR1 and FGFR3 (Fig. 1C). Neither expression of FGFR2 nor FGFR4 was detectable with western blotting under the conditions used in the present study. Genetic depletion of FGFRL1 had no obvious effects on the expression of other FGF receptors (Fig. 1C). Cell proliferation curves of those cells were comparable (Fig. 1D).

Effects of FGFRL1 gene depletion on tumour growth in a xenograft model. To examine the effects of FGFRL1 depletion in vivo, mice were injected subcutaneously with wild-type and FGFRL1-deficient KYSE520 cells. As presented in Fig. 2A and $\mathrm{B}$, tumours derived from FGFRL1-deficient cell lines (clones 15 and 21) exhibited reduced growth in vivo. The haematoxylin-eosin staining indicated that FGFRL1-deficient cells formed well-differentiated squamous cell carcinomas in a mouse xenograft model (Fig. 2C), whereas wild-type cells formed moderately differentiated squamous cell carcinomas, as originally described (19). These results indicated that FGFRL1 may contribute to tumorigenesis in vivo.

Effects of FGFRL1-depletion on mRNA expression profiles. In order to determine the underlying molecular mechanism of the deceleration of tumour growth resulting from FGFRL1-deficiency, mRNA levels were compared between wild-type and FGFRL1-deficient cells using microarrays (Fig.3A). Microarray demonstrated that the expression MMP-1,
C-X-C chemokine receptor type 7, keratin 14, FGFBP1 and keratin 6A was markedly downregulated in FGFRL1-deficient cells (Fig. 3B). In the five genes, reduced protein expression of MMP-1 and FGFBP1 were confirmed using western blotting (Fig. 3C).

MMP-1 digests collagen in a metal ion-dependent manner and is associated with cell invasion (20). Fig. 3D presents the invasion of cells through a collagen I membrane. The invasiveness of FGFRL1-deficient cells was decreased compared with that of wild-type KYSE520 cells.

FGFBP1 is a secreted FGF-binding protein that is able to promote cell motility (21). As presented in Fig. 3E and F, wild-type KYSE520 cells sealed a $400 \mu \mathrm{m}$ wide wound within 7 h, however, FGFRL1-deficient cells did not. Thus, FGFRL1-deficiency may have decreased the cell motility of KYSE520 cells compared with controls.

The structure of actin filaments was examined, since the filaments are required for cell motility (22). Visualization of actin filaments via phalloidin staining identified that actin filaments around the nucleus were sparse in FGFRL1-deficient cells compared with controls, whereas the filaments along the plasma membrane were observed as frequently as those of wild type cells (Fig. 3G). These results suggest that FGFRL1 may contribute to the regulation of actin filament assembly.

\section{Discussion}

While FGFRL1 expression tends to be high in ESCC patients with a poor prognosis (14), its function in the generation/expansion of ESCCs remains unresolved. In the present study, genetic depletion of FGFRL1 in ESCC KYSE520 cells indicated that 
A

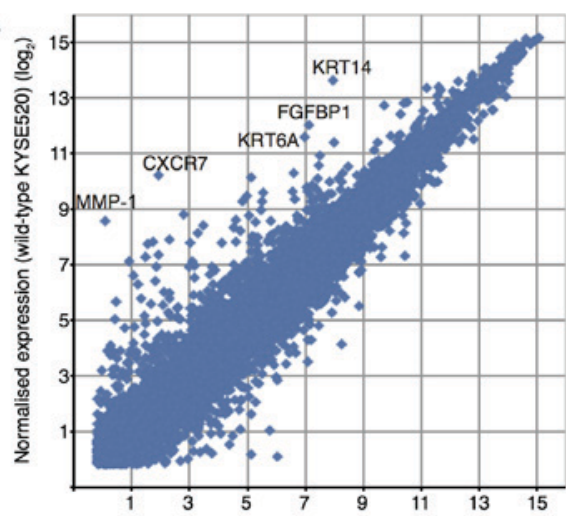

Normalised expression (FGFRL1-KO KYSE520) $\left(\log _{2}\right)$

\begin{tabular}{|r|r|r|r|}
\hline & \multicolumn{1}{|c|}{ Wild-type } & \multicolumn{1}{c|}{ KO } & \multicolumn{1}{c|}{ fold change } \\
\hline MMP-1 & 388.9129 & 1.0490 & 0.0027 \\
\hline CXCR7 & 1202.0803 & 3.8452 & 0.0032 \\
KRT14 & 12679.8217 & 249.1140 & 0.0196 \\
\hline FGFBP1 & 4206.1635 & 139.8964 & 0.0333 \\
\hline KRT6A & 3083.6335 & 125.7963 & 0.0408 \\
\hline
\end{tabular}

C

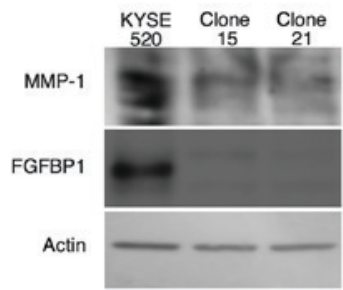

E
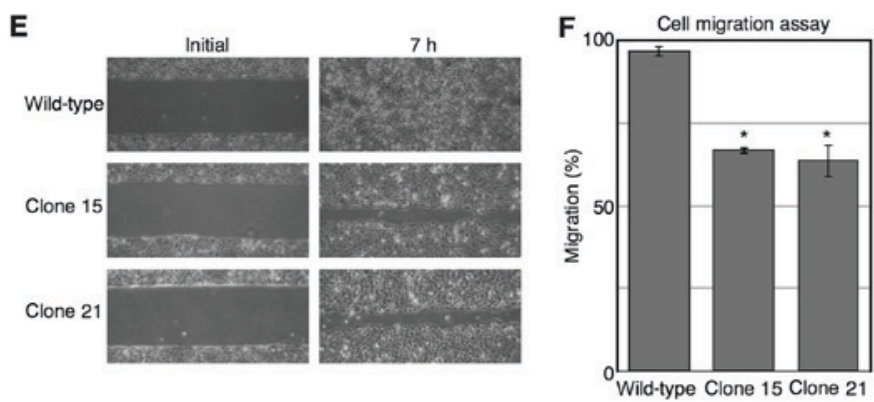

Wild-type Clone 15 Clone 21

G
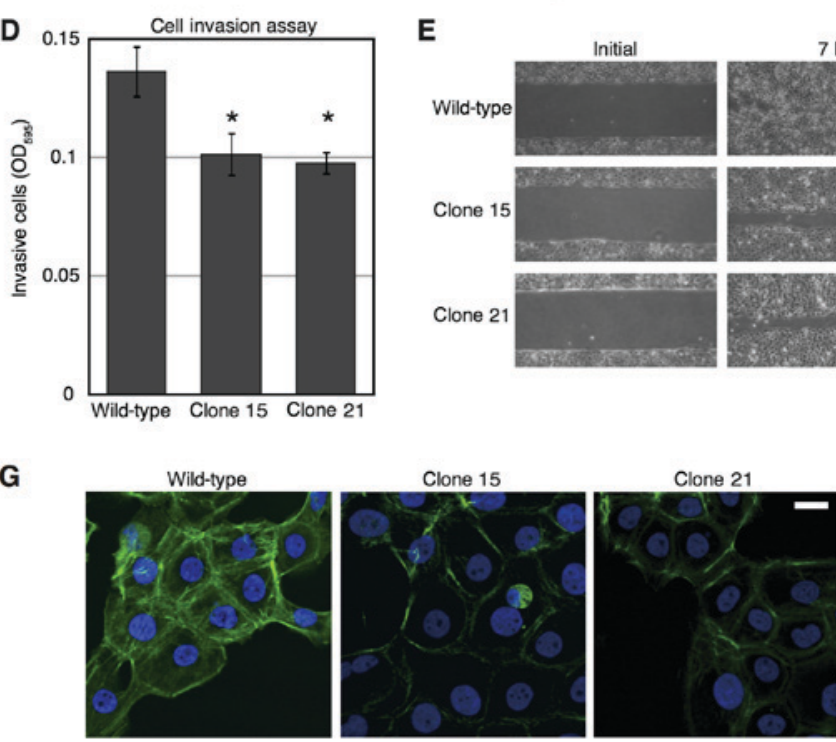

Figure 3. Microarray analysis of FGFRL1-deficient cells. (A) Identification of transcripts differentially expressed in wild-type and FGFRL1-deficient cells Mean of expression levels in parental KYSE520 cells and the clone 1, and those in clones 15 and 21, were plotted as 'wild-type KYSE520' and 'FGFRL1-KO KYSE520', respectively. Each dot represents an individual gene. Transcripts markedly downregulated in KYSE520-deficient cells are denoted by their gene symbol. (B) Genes exhibiting notably decreased expression in FGFRL1-deficient cells. (C) Western blot analysis of cell extracts prepared from the indicated cells. (D) Cell invasion through a porous type-I collagen membrane. After $24 \mathrm{~h}$, invaded cells were stained with a blue dye and permeabilised in $200 \mu \mathrm{l}$ of buffer. The experiment was repeated three times. Data represent the mean values. Error bars indicate the standard error of the mean. "P<0.05 (Dunnett's test) vs. the wild-type. (E) Cell migration assay. (F) The invaded area presented in (E) was assessed. The experiment was repeated three times. The data in the graph are expressed as mean values. Error bars indicate the standard error of the mean. " $\mathrm{P}<0.05$ (Dunnett's test) vs. the wild-type. (G) Phalloidin staining of cell lines. Scale bar, $10 \mu \mathrm{m}$. FGFRL1, fibroblast growth factor receptor-like 1; OD, optical density; MMP, matrix metalloproteinase; CXCR7, C-X-C chemokine receptor type 7; KRT14, keratin 14; FGFBP1, fibroblast growth factor binding protein 1; KRT6A, keratin 6A.

FGFRL1 may contribute to tumour growth in vivo. Tumour growth in a mouse xenograft model involves multiple processes, including cell proliferation, migration, adhesion and angiogenesis. Genetic depletion of the FGFRL1 gene failed to inhibit cell proliferation, although transient inhibition of FGFRL1 expression by siRNA induced cell cycle arrest $(13,16)$. This may suggest that FGFRL1-deficiency is able to affect cell proliferation transiently, but not permanently. This, in turn, suggests that genetic depletion of FGFRL1 is able to decrease cell motility and invasiveness, rather than cell proliferation.

In the present study, it was identified that FGFRL1-deficency decreased the expression level of proteins regulating cell motility and invasiveness, FGFBP1 and MMP-1. In vivo, FGFBP1 acts as a carrier protein that releases FGFs from the extracellular matrix, thereby increasing the proliferation, migration and angiogenesis induced by FGFs $(21,23)$. Consistently, the present study indicated that FGFRL1-deficiency reduced tumorigenic potential in a xenograft mouse model and decreased cell motility in vitro. Moreover, MMP-1 serves a critical function in local invasion of oesophageal carcinomas and is an independent prognostic factor (24). It was demonstrated in a previous study that high expression of FGFRL1 is associated with lymph node metastasis of ESCCs (14). In concurrence with those data, the present study demonstrated that FGFRL1-deficiency decreased the invasion of KYSE520 ESCC cells. Furthermore, it was demonstrated in the present study that actin filaments around the nucleus were sparse in FGFRL1-deficient cells. FGFRL1 may be able to promote tumour invasion via the regulation of protein expression and actin filament assembly, since the assembly is essential for motility and invasion of cells (22). The results of the present study suggest a reason for the association of FGFRL1 expression with lymph node metastasis of ESCCs. The underlying molecular mechanism by which FGFRL1 regulates the assembly of actin filaments remains unresolved.

The expression levels of MMP-1 and FGFBP1 were not decreased in normal tissues of FGFRL1-knockout 
mice $(10,12)$. The present results suggest that gene editing is useful for studying the function of a protein not only in normal development, but also in tumour generation/development.

To conclude, FGFRL1-knockout cell lines were established from ESCC KYSE520 cells. FGFRL1-deficiency decreased cell motility, invasion and tumorigenic potential of KYSE520 cells. FGFRL1 deficiency in KYSE520 cells also decreased the expression of proteins promoting cell motility and invasion, MMP-1 and FGFBP1. The present study may provide an explanation for the clinical observation that high expression of FGFRL1 in ESCCs is often associated with lymph node metastasis and poor prognosis of patients.

\section{Acknowledgements}

We would like to thank Ms. Takako Murai (Department of Nanobio Drug Discovery, Graduate School of Pharmaceutical Science, Kyoto University, Japan) for technical assistance.

\section{Funding}

The present study was supported by The Japan Society for the Promotion of Science [the Grant-In Aid for Scientific Research (B) (JSPS Kakenhi number 26293302)].

\section{Availability of data and materials}

The GEO accession number of the microarray results used in the present study is GSE96956. The datasets used and/or analysed during the current study are available from the corresponding author on reasonable request.

\section{Authors' contributions}

YT, KS and YS designed the study. YT, TM, KW and TS performed research. YT and YS analysed data and wrote the paper.

\section{Ethics approval and consent to participate}

All mice were handled and cared for in accordance with the Guide of Care and Use of Laboratory Animals, and all experiments were approved by the Ethics Committee of Experimental Animals of Kyoto University (Kyoto, Japan). All surgical procedures and postoperative care regimes were reviewed and approved by the Animal Care and Use Committee of Kyoto University.

\section{Consent for publication}

Not applicable.

\section{Competing interests}

The authors declare that they have no competing interests.

\section{References}

1. Ferlay J, Soerjomataram I, Dikshit R, Eser S, Mathers C Rebelo M, Parkin DM, Forman D and Bray F: Cancer incidence and mortality worldwide: Sources, methods and major patterns in GLOBOCAN 2012. Int J Cancer 136: E359-E586, 2015.
2. Thrift AP: The epidemic of oesophageal carcinoma: Where are we now? Cancer Epidemiol 41: 88-95, 2016.

3. Torre LA, Bray F, Siegel RL, Ferlay J, Lortet-Tieulent J and Jemal A: Global cancer statistics, 2012. CA Cancer J Clin 65: 87-108, 2015.

4. Tachimori Y, Ozawa S, Numasaki H, Ishihara R, Matsubara H, Muro K, Oyama $\mathrm{T}$, Toh $\mathrm{Y}$, Udagawa $\mathrm{H}$, Uno $\mathrm{T}$, et al: Comprehensive registry of esophageal cancer in Japan, 2010. Esophagus 14: 189-214, 2017.

5. Kuwano H, Nishimura Y, Oyama T, Kato H, Kitagawa $Y$, Kusano M, Shimada H, Takiuchi H, Toh Y, Doki Y, et al: Guidelines for diagnosis and treatment of carcinoma of the esophagus April 2012 edited by the Japan Esophageal Society. Esophagus 12: 1-30, 2015.

6. Wiedemann $\mathrm{M}$ and Trueb $\mathrm{B}$ : Characterization of a novel protein (FGFRL1) from human cartilage related to FGF receptors. Genomics 69: 275-279, 2000.

7. Stauber DJ, DiGabriele AD and Hendrickson WA: Structural interactions of fibroblast growth factor receptor with its ligands. Proc Natl Acad Sci USA 97: 49-54, 2000.

8. Trueb B: Biology of FGFRL1, the fifth fibroblast growth factor receptor. Cell Mol Life Sci 68: 951-964, 2011.

9. Gerber SD, Steinberg F, Beyeler M, Villiger PM and Trueb B: The murine Fgfrl1 receptor is essential for the development of the metanephric kidney. Dev Biol 335: 106-119, 2009.

10. Gerber SD, Amann R, Wyder S and Trueb B: Comparison of the gene expression profiles from normal and Fgfrl1 deficient mouse kidneys reveals downstream targets of Fgfrl1 signaling. PLoS One 7: e33457, 2012.

11. Baertschi S, Zhuang L and Trueb B: Mice with a targeted disruption of the Fgfrll gene die at birth due to alterations in the diaphragm. FEBS J 274: 6241-6253, 2007.

12. Amann R, Wyder S, Slavotinek AM and Trueb B: The FgfrL1 receptor is required for development of slow muscle fibers. Dev Biol 394: 228-241, 2014.

13. Tsuchiya S, Fujiwara T, Sato F, Shimada Y, Tanaka E, Sakai Y, Shimizu K and Tsujimoto G: MicroRNA-210 regulates cancer cell proliferation through targeting fibroblast growth factor receptor-like 1 (FGFRL1). J Biol Chem 286: 420-428, 2011.

14. Shimada Y, Okumura T, Nagata T, Hashimoto I, Sawada S, Yoshida T, Fukuoka J, Shimizu K and Tsukada K: Expression analysis of fibroblast growth factor receptor-like 1 (FGFRL1) in esophageal squamous cell carcinoma. Esophagus 11: 48-53, 2014.

15. Shimada Y, Okumura T, Takei Y, Watanabe K, Nagata T, Hori T, Tsuchiya S, Tsukada K and Shimizu K: Role of fibroblast growth factor receptors in esophageal squamous cell carcinoma. Esophagus 13: 30-41, 2016.

16. Zuo J, Wen M, Lei M, Peng X, Yang X and Liu Z: MiR-210 links hypoxia with cell proliferation regulation in human Laryngocarcinoma cancer. J Cell Biochem 116: 1039-1049, 2015.

17. Schild C and Trueb B: Aberrant expression of FGFRL1, a novel FGF receptor, in ovarian tumors. Int J Mol Med 16: 1169-1173, 2005.

18. Donnard E, Asprino PF, Correa BR, Bettoni F, Koyama FC, Navarro FC, Perez RO, Mariadason J, Sieber OM, Strausberg RL, et al: Mutational analysis of genes coding for cell surface proteins in colorectal cancer cell lines reveal novel altered pathways, druggable mutations and mutated epitopes for targeted therapy. Oncotarget 5: 9199-9213, 2014.

19. Shimada Y, Imamura M, Wagata T, Yamaguchi $\mathrm{N}$ and Tobe T: Characterization of 21 newly established esophageal cancer cell lines. Cancer 69: 277-284, 1992.

20. Pulukuri SM and Rao JS: Matrix metalloproteinase-1 promotes prostate tumor growth and metastasis. Int J Oncol 32: 757-765, 2008.

21. Tassi E, McDonnell K, Gibby KA, Tilan JU, Kim SE, Kodack DP Schmidt MO, Sharif GM, Wilcox CS, Welch WJ, et al: Impact of fibroblast growth factor-binding protein-1 expression on angiogenesis and wound healing. Am J Pathol 179: 2220-2232, 2011.

22. Bugyi B and Carlier MF: Control of actin filament treadmilling in cell motility. Annu Rev Biophys 39: 449-470, 2010.

23. Abuharbeid S, Czubayko F and Aigner A: The fibroblast growth factor-binding protein FGF-BP. Int J Biochem Cell Biol 38: 1463-1468, 2006.

24. Yamashita K, Mori M, Kataoka A, Inoue H and Sugimachi K: The clinical significance of MMP-1 expression in oesophageal carcinoma. Br J Cancer 84: 276-282, 2001.

This work is licensed under a Creative Commons Attribution-NonCommercial-NoDerivatives 4.0 International (CC BY-NC-ND 4.0) License. 